\title{
VASCULARITY CHANGE AND TUMOR RESPONSE TO NEOADJUVANT CHEMOTHERAPY FOR ADVANCED BREAST CANCER
}

\author{
Wen-Hung Kuo, ${ }^{* \dagger}$ Chiung-Nien Chen, ${ }^{* * *}$ Fon-Jou Hsien, ${ }^{\dagger}{ }^{\dagger}$ MI Ming-Kwang Shyu, ${ }^{\S}$ \\ Li-Yun Chang, ${ }^{\S}$ Po-Huang Lee, ${ }^{* \dagger}$ Li-Yu D. Liu,, Chia-Hsien Cheng, ${ }^{*} J_{\text {Ane Wang, }}$ ** \\ and KING-Jen ChANG*' \\ *Department of Surgery, National Taiwan University Hospital; ${ }^{\dagger}$ Angiogenesis Research Center, National Taiwan \\ University; ${ }^{*}$ Division of Mechanics, Research Center for Applied Sciences, Academia Sinica; ${ }^{\S}$ Department of \\ Obstetrics and Gynecology, National Taiwan University Hospital; ${ }^{\text {II }}$ Graduate Institute of Clinical Research, College \\ of Medicine, National Taiwan University; "Department of Agronomy, Biometrics Division, National Taiwan \\ University; "Department of Oncology, National Taiwan University Hospital; and ** Department of Medical Imaging, \\ National Taiwan University Hospital, Taipei, Taiwan
}

\section{(Received 29 March 2007; revised 1 November 2007; in final form 9 November 2007)}

\begin{abstract}
For advanced breast cancer with severe local disease (ABC) (stage III/IV), neoadjuvant chemotherapy improves local control and surgical outcome. However, about $\sim 20$ to $30 \%$ of advanced cancers show either no or poor response to chemotherapy. To prevent unnecessary treatment, a capability of predicting clinical response to neoadjuvant chemotherapy of $\mathrm{ABC}$ is highly desirable. Vascularity index (VI) of breast cancers was derived from the quantification results in $30 \mathrm{ABC}$ patients by using power Doppler sonography. Power Doppler sonography evaluation was performed every one to two weeks during chemotherapy. The overall response rate for $\mathbf{3 0}$ advanced patients tested was $\mathbf{7 0 \%}$, when $\mathbf{5 0 \%}$ or more reduction in tumor size was the objective clinical response. Chemotherapy response was unrelated to the original tumor size $(p=\mathbf{0 . 5 6 3})$ or chemotherapy agents used $(p=0.657)$. The median VI for all 30 patients was $4.99 \%$. The response rates for hypervascular tumors vs. hypovascular tumors, based on initial median value, were $86.7 \%$ and $53.3 \%$, respectively $(p=0.109)$. The average VIs in responders and nonresponders were $7.67 \pm 4.77 \%$ and $4.01 \pm 3.82 \%(p=0.052)$. There was a tendency for responders who have a relatively high initial vascularity. The VI change in responder group shows a pattern of initial increasing in vascularity followed by decreasing in vascularity. All patients (17/17) with a VI increment of $>5 \%$ during chemotherapy had good chemotherapy response, whereas in patients with a VI increment of $<5 \%$, the response rate was $30.8 \%(4 / 13)(p<$ 0.001). For patients with a peak VI of $>10 \%$ during chemotherapy, the response rate was $94.1 \%(16 / 17)$. However, in patients with a peak VI of $<10 \%$, the response rate was $38.5 \%(5 / 13)(p=0.001)$. This prediction was made mostly within one month $(25.47 \pm 12.96 \mathrm{~d}$ for VI increments $>5 \%$ and $25.44 \pm 12.41 \mathrm{~d}$ for VI increased to $>10 \%)$. In the meantime, the differences in size reduction shown in B-mode sonography were insignificant between responders and nonresponders (patient group with VI increment $>5 \%, p=0.308$; patient group with peak $\mathrm{VI}>10 \%, p=0.396$ ). In conclusion, we propose that VI as determined by using power Doppler sonography is a good and inexpensive clinical tool for monitoring vascularity changes during neoadjuvant chemotherapy in ABC patients. Two parameters-VI increment $>5 \%$ and peak VI $>10 \%$ - are potential early predictors for good responses to neoadjuvant chemotherapy within one month in patients with ABC. (E-mail: kingjen@ha.mc.ntu.edu.tw) @ 2008 Published by Elsevier Inc. on behalf of the World Federation for Ultrasound in Medicine \& Biology.
\end{abstract}

Key Words: Advanced breast cancer (ABC), Angiogenesis, Increasing in vascularity, Neoadjuvant chemotherapy, Power Doppler sonography, Vascularity index (VI).

\section{INTRODUCTION}

Most breast cancers are thought to be systemic disease at diagnosis (Bonadona et al. 1991; Smith et al. 1991). Adju-

Address correspondence to: King-Jen Chang, M.D., Ph.D., Professor., Department of Surgery, National Taiwan University Hospital, No. 7, Chung-Shan South Road, 100 Taipei, Taiwan. E-mail: kingjen@ha.mc.ntu.edu.tw vant chemotherapy is necessary for $80 \%$ of patients in Taiwan and can substantially improve disease-free and overall survival. For advanced breast cancer (ABC) with severe local disease (stage III/IV), preoperative chemotherapy improves local control and surgical outcome. About 20 to $30 \%$ of $\mathrm{ABCs}$ show either no or poor response to chemotherapy (Bonadona et al. 1991; Smith et al. 1991). To this subgroup of patients, early prediction of tumors in 
response to neoadjuvant chemotherapy is desirable. In this report, we intend to address a clinical diagnosis tool in monitoring the efficacy of neoadjuvant chemotherapy for $\mathrm{ABC}$. This is important to both save money and spare patients' suffering during ineffective therapy.

Increased tumor vascularity may be a surrogate marker for prediction of tumor response to chemotherapy (Cosgrove et al. 1990, 1993). The research evidence shows that tissue necrosis induced by cytotoxic agents would release many kinds of angiogenic factors (Shaked et al. 2006). Increased vascularity is essential for the growth and metastasis of solid tumors (Folkman et al. 1963). Several studies have reported that increased tumor vascularity is an independent prognostic indicator in primary breast carcinoma (Toi et al. 1993; Weidner et al. 1991, 1992). Another possible mechanism was found in animal model that chemotherapy induces acute recruitment of bone marrow-derived circulating endothelial progenitor cells to tumors to enhance tumor vascularity (Shaked et al. 2006).

With Doppler sonography, tumor vascularity can be assessed in vivo (Dixon et al. 1992). Doppler sonography showed high sensitivity for predicting complete histologic response (Caruso et al. 1995; Lagalla et al. 1998; Singh et al. 2005). After neoadjuvant chemotherapy, Doppler sonography was more sensitive in disclosing the presence of active neoplastic tissue than conventional histopathological results (Huber et al. 2000; Vallone et al. 2005). In previous reports (Kedar et al. 1994; Roberrto et al. 1998), decreased tumor vascularity at the end of treatment indicates good response, whereas increased or unchanged vascularity indicates no response. In $40 \%$ of these patients, the Doppler changes appeared four weeks before a size reduction was detectable using B-mode ultrasonography (Kedar et al. 1994). Thus, Doppler flow imaging can be helpful in both assessing and predicting the response of breast cancer to medical treatment.

Power Doppler sonography has shown a higher sensitivity in the detection of blood flow than conventional color Doppler sonography (Martinoli et al. 1998). It can be used as a tool for quantification of tumor vascularity via the color pixels of vessels scanned by power Doppler ultrasound (Cosgrove et al. 1990, 1993). Vascularity index (VI) is an ultrasound parameter for quantitatively evaluating in vivo increased vascularity. It offers the advantage of covering the entire tumor section for signal acquisition, i.e., avoiding the signal bias may be from selected portions of tumor section. Consequently, the color signal in a given tumor section could be quantified. One of our reports has pointed out that the vascularity index could be used to differentiate the nature of neck lymph nodes (Wu et al. 1998). It has a good correlation with status of lymph node metastasis in cervical carcinoma (Cheng et al. 1999). It is also a good preoperative indicator of recurrence and patient survival in colon cancer (Chen et al. 2000). In addition, it is an indicator of the efficacy of thalidomide in unresectable hepatocellular carcinoma (Hsu et al. 2005). The aim of this study is to evaluate the usefulness of VI of power Doppler sonography as a predictor for tumor response to neoadjuvant chemotherapy in locally advanced breast cancer. This will allow clinicians to consider the modification of the treatment protocol at the early stage of chemotherapy based on Doppler findings.

\section{MATERIALS AND METHODS}

\section{Patients and chemotherapy}

Thirty consecutive ABC patients (TNM stage IIB, III and IV), who received preoperative chemotherapy for tumor reduction or disease control from June 21, 2000 until June 3, 2002 at National Taiwan University Hospital (NTUH), were recruited for this study. The diagnosis of $\mathrm{ABC}$ was made according to the American Joint Committee on Cancer (AJCC)'s definition of breast cancer staging. These patients consisted of two at stage IIB, 19 at stage III and nine at stage IV, respectively. Eight patients had T4 disease documented by the pathologists and one patient with inflammatory breast cancer (T4 days) was included.

Intravenous chemotherapy was given for three weeks per cycle in these patients. The first seventeen patients who joined the Paclitaxel-based neoadjuvant chemotherapy clinical trial, received Paclitaxel (Phyxol, Sinphar Pharmaceutical, Taipei, Taiwan) $175 \mathrm{mg} / \mathrm{m}^{2}$ by 3-h IV infusion immediately followed by cisplatin 50 $\mathrm{mg} / \mathrm{m}^{2}$ by $24-\mathrm{h}$ IV infusion on day 1 of a three-week cycle. Eleven patients received Epirubicin (Pharmorubicin, Pfizer Pharmaceuticals, New York City, NY, USA) $90 \mathrm{mg} / \mathrm{m}^{2}$, cyclophosphamide $600 \mathrm{mg} / \mathrm{m}^{2}$ and 5-Fluorouracil $500 \mathrm{mg} / \mathrm{m}^{2}$ on day 1 every three weeks. The remaining two stage IV patients received Vinorelbine (Navelbine, Glaxo Wellcome, Inc., Research Triangle Park, $\mathrm{NC}$, USA) $25 \mathrm{mg} / \mathrm{m}^{2}$ on day 1 and day 8 as chemotherapy regimen based on patient's choice. All patients gave informed consent according to guidelines approved by the Institutional Review Board of NTUH.

\section{Sonography evaluation}

For each case, power Doppler sonography (HDI 5000; Advanced Technology Laboratories, Bothell, WA, USA) with 5- to $12-\mathrm{MHz}$ broad band linear array transducers (Transducer model: L12-5 $50 \mathrm{~mm}$ Broadband Linear Array, multifocal zone, 8 transmit zones), along with conventional 2-D sonography were performed before incision biopsy, on day 8 and day 15 during therapy and/or before mastectomy. 


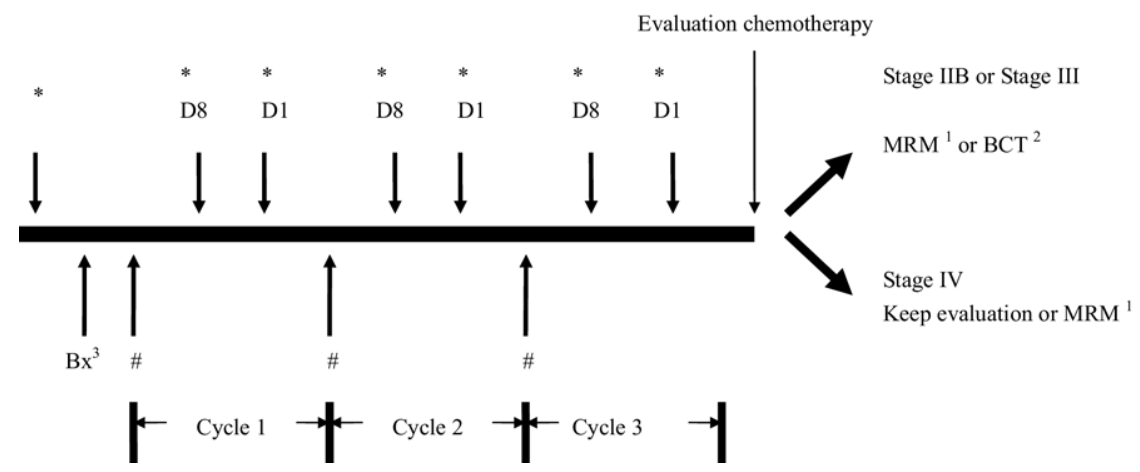

Fig. 1. Chemotherapy and evaluation protocol. $*$ Sonography evaluation; \#chemotherapy was given on day $1 ;{ }^{1} \mathrm{MRM}=$ Modified radical mastectomy; ${ }^{2} \mathrm{BCT}=$ Breast conserving treatment; ${ }^{3} \mathrm{Bx}=$ incision biopsy.

\section{Evaluation of chemotherapy response}

Tumor response was decided after three cycles of chemotherapy in all patients (i.e., 9 to 12 weeks after the initiation of chemotherapy). At the time of decisive evaluation, the clinical response was classified as complete response (CR), partial response (PR), stable disease (SD) or progressive disease (PD) according to standard Union International Contre le Cancer (UUIC) criteria (Hayward et al. 1977). CR was defined as the disappearance of all clinical evidence of the tumor including the axillary site. PR was defined as a reduction of $50 \%$ or more in the cross section area on B-mode sonography, without the appearance of new lesions. SD was defined as a decrease of $<50 \%$ in the cross section area or an increase of $<25 \%$, without the appearance of new lesions. Any measured or estimated increase $>25 \%$ or the appearance of new lesions was defined as PD. The patients were classified into two groups: the responder, who was classified as CR or $\mathrm{PR}$, and the nonresponder, who was classified as SD or PD. All these data and other clinical information were analyzed, integrated and correlated.

Sonographic technique and quantification of vascular density in power Doppler images

The scanner used was a power Doppler ultrasound HDI 5000 model (Advanced Technology Laboratories). Settings of the power Doppler sonography were standardized for the highest sensitivity in the absence of apparent noise. They were standardized with a medium wall filter, pulsed repetition at frequency of $1000 \mathrm{~Hz}$, moderate-to-long persistence and a slow sweep technique (a technique for small vessels within breast tumor detection. We recorded the Doppler signals of them after the signals became maximized and stabilized that required a slow mode in scanning the signal using the standard transducer of HDI 5000 model). Under these conditions, the lowest possible measurable velocity was claimed below $5 \mathrm{~cm} / \mathrm{s}$. Focusing depth was set between 0.5 and $4 \mathrm{~cm}$. Each tumor was scanned thoroughly, and a tangential scanning was made to avoid the nonrepresentative section. The power Doppler window was set to cover the whole tumor on the screen. If the maximal tumor diameter was larger than the width of sonography probe, the chosen power Doppler window should be filled with tumor and show partial image of the tumor. The tumor section was then scanned carefully in all directions. To avoid section bias, we recorded sections with possible maximal power Doppler signals. At least three sections were recorded during each evaluation. They were quantified after evaluation and the tumor section with subjectively maximal power Doppler signals was captured and stored for later analysis. Each tumor was scanned at least three times. These tumor sections with maximal power Doppler signals were available for quantitative analysis. After the examination, the previously stored images were retrieved on the monitor. The tumor margin was contoured using a cursor. Quantification of the vascular color signals within the demarcated tumor area was then automatically

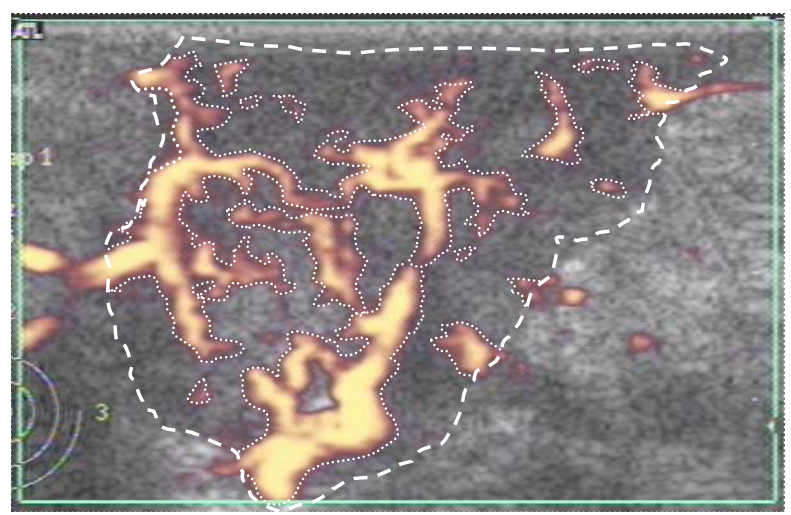

Fig. 2. Definition of vascularity index (VI) $=$ the number of colored pixels within the tumor section (area defined by fine dots)/the number of total pixels in that particular tumor section (area defined by bigger dots). 

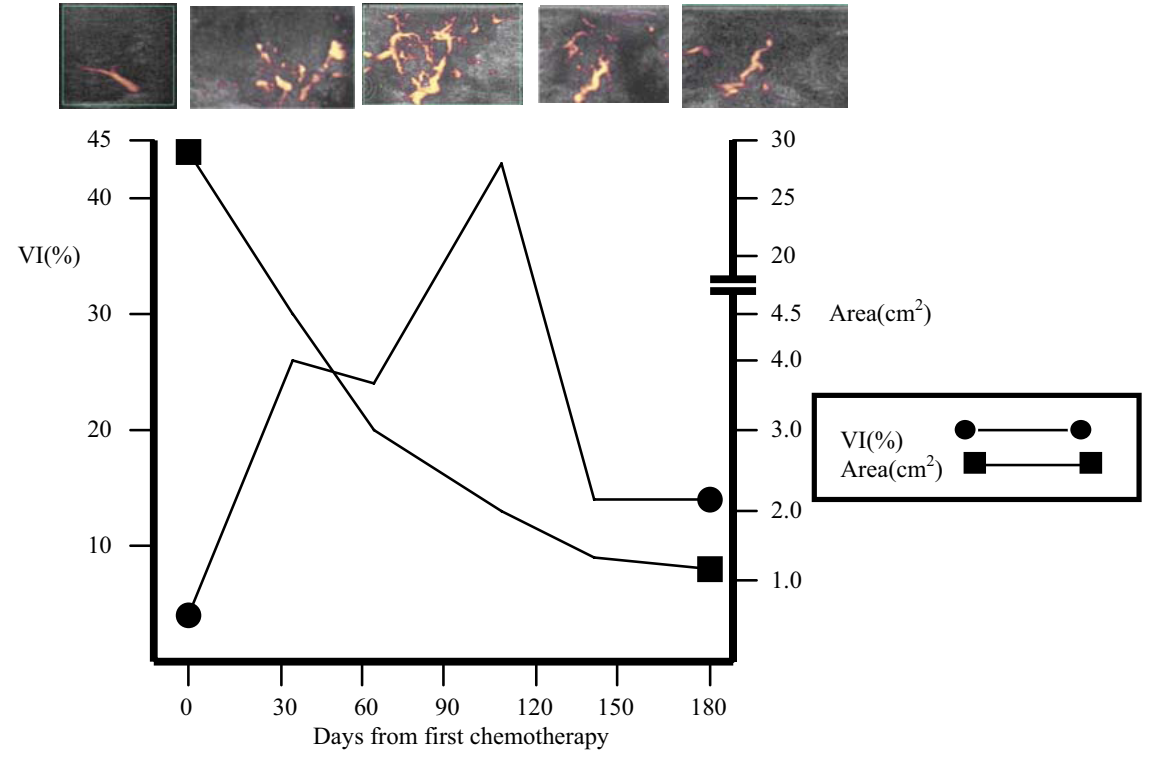

(a)
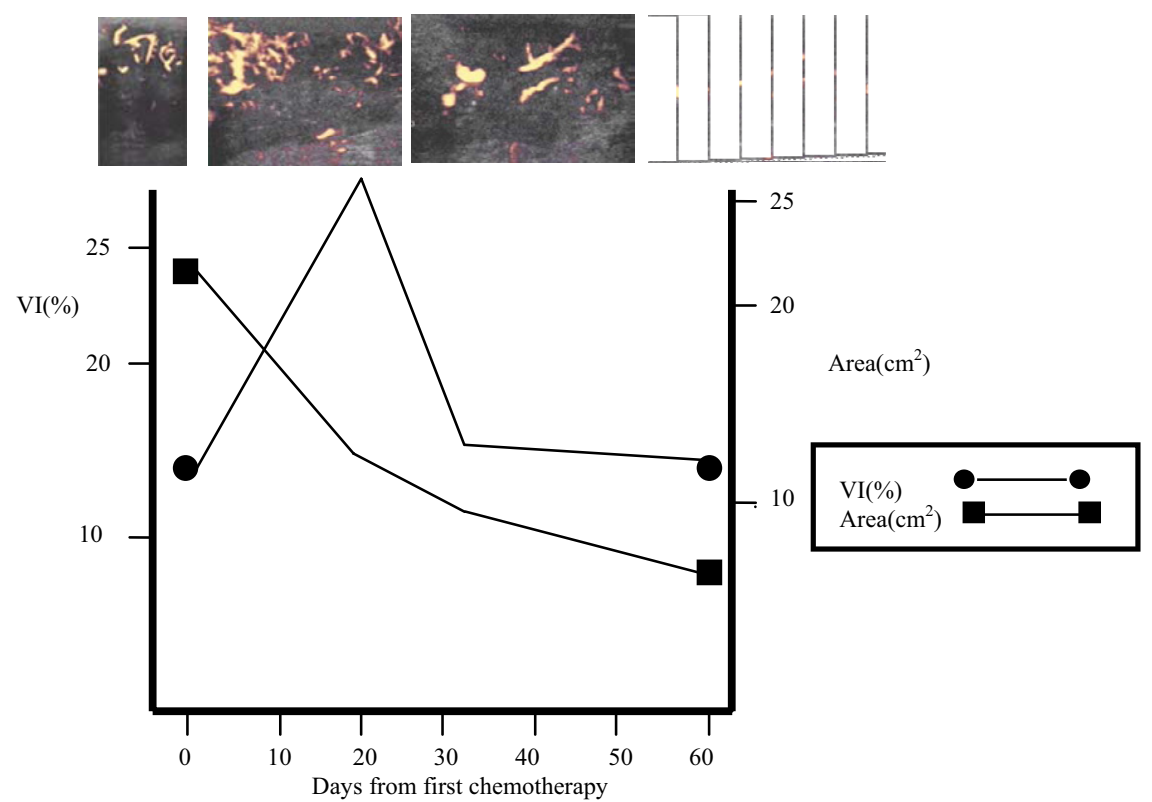

(b)

Fig. 3. The typical pattern of vascularity index change and tumor size reduction during chemotherapy for responders. (a) The patient with initial VI less than median $4.99 \%$ (hypovascular tumor). (b) The patient with initial VI greater than median $4.99 \%$ (hypervascular tumor). Vascularity index $(\mathrm{VI})=$ the number of colored pixels within the tumor section/the number of total pixels in that particular tumor section.

performed by special software (Encomate; Electronic Business Machine Co., Ltd., Taipei, Taiwan). The results were expressed as the VI (the number of colored pixels within the tumor section/the number of total pixels in that particular tumor section). For each tumor, the maximum of the VI of representative tumor sections was used for statistical analysis. VI increment was defined as the change from baseline value to peak VI. More detailed description of VI quantification has been documented (Chen et al. 2000 and 2005).

\section{Data analysis}

Continuous variables were described as mean \pm standard deviation. The nominal variables were demonstrated as frequency and percentage. Patients were grouped according to their VI status, tumor size and treatment regimen. The difference between two groups was tested by using Student's $t$-test for the variable with interval scale, or by using Fisher's exact test for the variable with nominal scale. All analyses were per- 

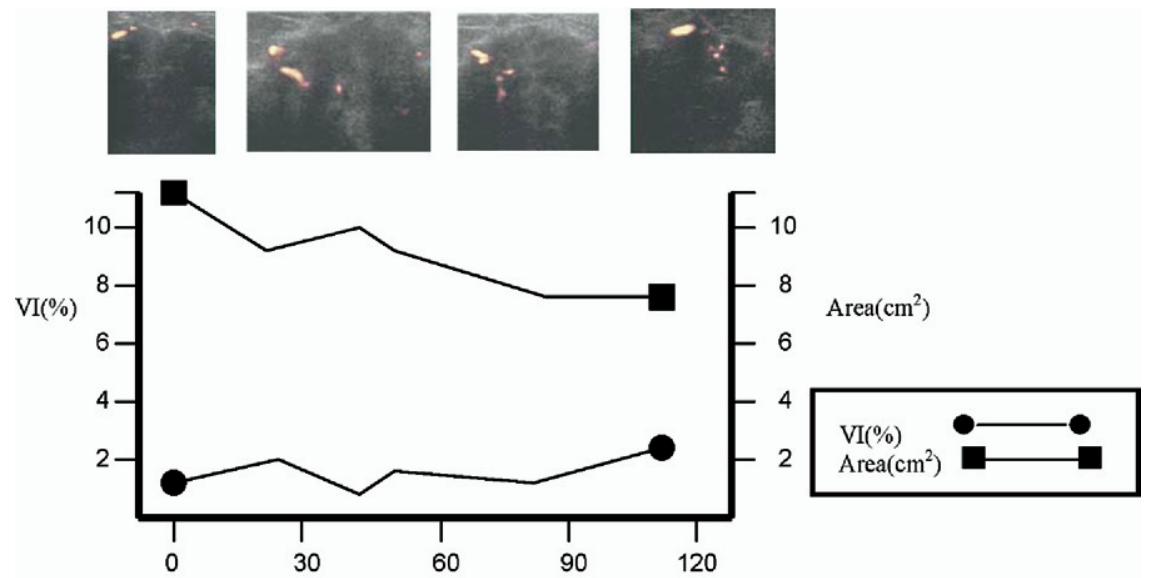

(a)

Days from first chemotherapy

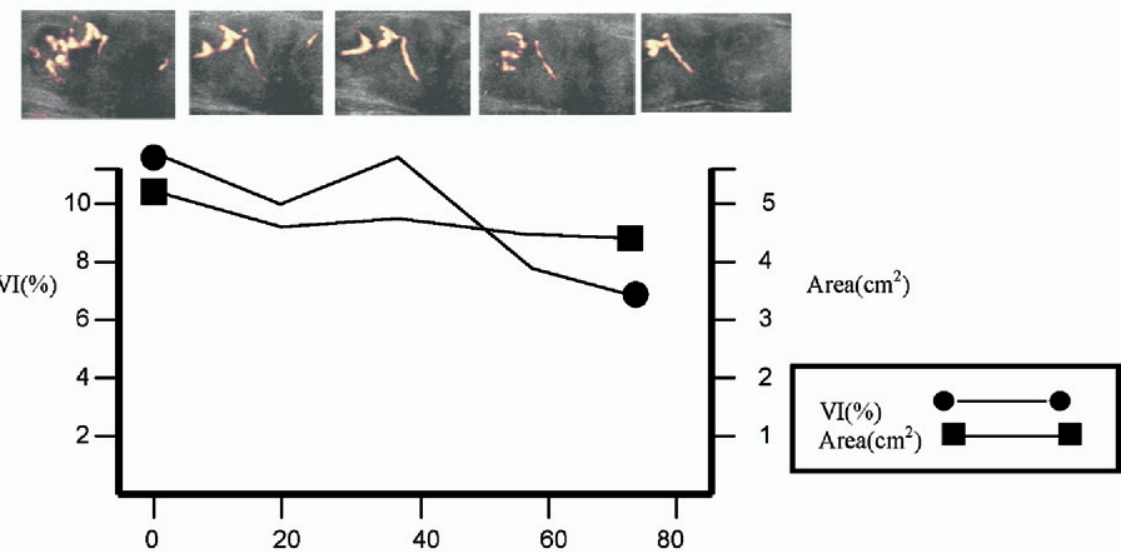

(b)

Days from first chemotherapy

Fig. 4. The typical pattern of VI variation and tumor size reduction during chemotherapy for nonresponders. (a) The patient with initial VI less than median $4.99 \%$ (hypovascular tumor). (b) The patient with initial VI greater than median $4.99 \%$ (hypervascular tumor). Vascularity index $(\mathrm{VI})=$ the number of colored pixels within the tumor section/the number of total pixels in that particular tumor section.

formed using SAS software, version 9.1 (SAS Institute, Inc., Cary, NC, USA). Statistical significance was set at $\alpha=0.05$ level for all analyses.

\section{RESULTS}

Sonographic evaluation was performed every one to two weeks during chemotherapy according to the stan- dard protocol for the clinical trial of chemotherapy (Fig. 1). The quantitative measurement for vascularity in tumor is shown in Fig. 2. Among 30 patients, 21 were responders to chemotherapy, eight remained stable in their disease and one developed progressive disease. The overall response rate was $70 \%$. The mean VI of these 30 cases was $6.57 \pm 6.63 \%$. The medium VI was $4.99 \%$.

Table 1. Chemotherapy response in related to VI change during neoadjuvant chemotherapy

\begin{tabular}{lccccc}
\hline \multicolumn{1}{c}{ Feature } & & No. of patients (\%) & Responder & Non-responder & $p$-value (Fisher) \\
\hline No. of patients (in \%) & & $30(100.0 \%)$ & $21(70.0 \%)$ & $9(30.0 \%)$ & \\
VI Increment & $>5 \%$ & $17(56.7 \%)$ & $17(100.0 \%)$ & $0(0.0 \%)$ & $<0.001$ \\
& $\leqq 5 \%$ & $13(43.3 \%)$ & $4(30.8 \%)$ & $9(69.2 \%)$ & 0.002 \\
Peak VI & $>10 \%$ & $17(56.7 \%)$ & $16(94.1 \%)$ & $1(5.8 \%)^{*}$ & $8(61.5 \%)$ \\
& $\leqq 10 \%$ & $13(43.3 \%)$ & $5(38.5 \%)$ & 0 & \\
\hline
\end{tabular}

Vascularity index $(\mathrm{VI})=$ the number of colored pixels within the tumor section/the number of total pixels in that particular tumor section.

* VI decreased after chemotherapy. 
Table 2. The average time interval needed to get $5 \%$ VI increment or $>10 \% \mathrm{VI}$ in chemotherapy responders

\begin{tabular}{lcc}
\hline $\begin{array}{c}\text { VI measured after } \\
\text { neoadjuvant } \\
\text { chemotherapy }\end{array}$ & $\begin{array}{c}\text { No. of } \\
\text { Responders }\end{array}$ & $\begin{array}{c}\text { Time interval required } \\
\text { for tumor } \\
\text { VI increment }\end{array}$ \\
\hline $\begin{array}{l}\text { VI increment }>5 \% \\
\text { Peak VI }>10 \%\end{array}$ & 17 & $25.47 \pm 12.96 \mathrm{~d}$ \\
\hline
\end{tabular}

Vascularity index $(\mathrm{VI})=$ the number of colored pixels within the tumor section/the number of total pixels in that particular tumor section.

* Nine patients with original VI $<10 \%$.

The typical vascularity change in chemotherapy responder shows a pattern of initially increasing in vascularity followed by decreasing in vascularity (Fig. 3). The VI profile in nonresponders did not show evident fluctuation (Fig. 4). All patients (17/17) with a VI increment of $>5 \%$ during chemotherapy had good chemotherapy response, whereas in patients with a VI increment of $<5 \%$, the response rate was $30.8 \%(4 / 13)(p<0.001)$ (Table 1). In patients showing a peak of VI $>10 \%$ during chemotherapy, the response rate was $94.1 \%(16 / 17)$. However, in patients with a peak VI of $<10 \%$, the response rate was $38.5 \%(5 / 13)(p=0.002)$ (Table 1$)$.

In 17 patients with a VI increment $>5 \%$, the average time interval required to reach this cutoff point is $25.47 \pm 12.96 \mathrm{~d}$. The size reduction in cross section area at this time point was $27.35 \pm 18.34 \%$. For nine responsive patients with original VI of $<10 \%$, their VI increased to $>10 \%$ around $25.44 \pm 12.41$ days after chemotherapy. The size reduction at this time point was $26.19 \pm 18.28 \%$ (including all 16 responders). In nonresponsive patients, the size reduction on day $25 \pm 5$ after the first course of chemotherapy is $20.44 \pm 10.15 \%$. At this time point, the size reduction in cross section area between responders with a VI increment of $>5 \%$ and nonresponders showed no statistical significance $(p=$ 0.308). The size reduction between patients with a peak VI of $>10 \%$ and nonresponders showed no statistical significance either $(p=0.396)$ (Tables 2 and 3).

The effects of both original tumor properties and chemotherapy agents to chemotherapy response among ABC patients were also analyzed (Table 4). There was no significance in the response rates between both responder and nonresponder when they were grouped into hypervascular and hypovascular tumors based on the initial median VI $(p=0.109)$. Mean original VI between responders and nonresponders were different but not significant $(p=0.052)$ (Table 5). The estimated corresponding cut surface area for tumor with its largest diagonal diameter of $3 \mathrm{~cm}$ was $7.06 \mathrm{~cm}^{2}(1.5 \mathrm{~cm} \times 1.5$ $\mathrm{cm} \times \pi=7.06 \mathrm{~cm}^{2}$ ). The response rates in larger tumors $\left(\right.$ area $>7 \mathrm{~cm}^{2}$ ) and smaller tumors (area $<7 \mathrm{~cm}^{2}$ ) were
$73.6 \%$ and $63.6 \%$, respectively $(p=0.687)$. Seventeen patients received neoadjuvant chemotherapy with Paclitaxel; the response rate was $64.7 \%$. The response rate of patients who received Epirubicin-based treatment was 72.7\%. Two patients received Vinorelbine as neoadjuvant agent and had partial response. The response rates between patients receiving Paclitaxel or Epirubicinbased neoadjuvant chemotherapy showed no statistical difference $(p=0.999)$ (Table 4).

\section{DISCUSSION}

Over the last decade, the use of neoadjuvant (preoperative) chemotherapy has emerged as the standard of care for patients with locally advanced breast caner. However, two large randomized trials proved that the survival rates were equivalent in patients receiving preoperative or postoperative chemotherapy (Clahsen et al. 1996; Mamounas 1998). The shortcoming of current clinical practice is time consuming because the decision making is based on the pathologic response at the end of chemotherapy.

This is the first study that shows the role of power Doppler in monitoring neoadjuvant chemotherapeutic response. Therefore, it may shorten the time required for alternative therapeutic decision making when a subset of locally advanced breast cancer has poor response to certain chemotherapy strategies.

The mean VI of these 30 cases was $6.57 \pm 6.63 \%$. The median VI was $4.99 \%$. The overall response rate was $70 \%$.

This response rate was compatible with other reported series (Smith et al. 2002). With quantitative measurement and sequential observation, we observed the vascularity change during chemotherapy of $\mathrm{ABC}$. We have noticed that the typical trajectory of VI in chemotherapy responder revealed increasing vascularity with

Table 3. The percentage of size reduction in cross section area on day $25 \pm 5$ after first course of chemotherapy in responders whose VI increment was $>5 \%$, responders whose peak VI was $>10 \%$ and nonresponders

\begin{tabular}{lccc}
\hline \multicolumn{1}{c}{ Feature } & $\begin{array}{c}\text { No. of } \\
\text { patients }\end{array}$ & $\begin{array}{c}\text { Tumor size reduction (\%) in } \\
\text { cross section area on day 25 }\end{array}$ \\
\hline VI increment $>5 \%$ & 17 & $27.35 \pm 18.34 \%$ & $p=0.308^{*}$ \\
Peak VI $>10 \%$ & 16 & $26.19 \pm 18.28 \%$ & $p=0.396 \dagger$ \\
Nonresponders & 9 & $20.44 \pm 10.15 \%$ & \\
\hline
\end{tabular}

Vascularity index $(\mathrm{VI})=$ the number of colored pixels within the tumor section/the number of total pixels in that particular tumor section.

* Comparison of size reduction percentage between responders whose VI increment was $>5 \%$ and nonresponders.

$\dagger$ Comparison of size reduction percentage between responders whose peak VI was $>10 \%$ and nonresponders. 
Table 4. The statistical evaluation of the differential effects on chemotherapy responses in ABC patients based on initial VI, original tumor section area and chemotherapy agents, respectively

\begin{tabular}{|c|c|c|c|c|c|}
\hline \multicolumn{2}{|c|}{ Feature } & No. of patients $(\%)$ & Responder & Nonresponder & $\begin{array}{l}p \text {-value } \\
\text { (Fisher) }\end{array}$ \\
\hline \multicolumn{2}{|c|}{ The number of patients(in percentage) } & $30(100 \%)$ & $21(70 \%)$ & $9(30 \%)$ & \\
\hline \multirow[t]{2}{*}{ Initial VI* } & Hypervascularity* & $15(50.0 \%)$ & $13(86.7 \%)$ & $2(13.3 \%)$ & 0.109 \\
\hline & Hypovascularity & $15(50.0 \%)$ & $8(53.3 \%)$ & $7(46.7 \%)$ & \\
\hline \multirow[t]{3}{*}{ Original tumor section area } & $>7 \mathrm{~cm} \dagger$ & $19(63.3 \%)$ & $14(73.7 \%)$ & $5(26.3 \%)$ & 0.687 \\
\hline & $\leqq 7 \mathrm{~cm}^{2}$ & $11(37.8 \%)$ & $7(63.6 \%)$ & $4(36.4 \%)$ & \\
\hline & & & & & \multirow{4}{*}{$\begin{array}{l}\text { Paclitaxel vs. Epirubicin } \\
0.999\end{array}$} \\
\hline \multirow[t]{3}{*}{ Chemotherapy agent } & Paclitaxel & $17(56.6 \%)$ & $11(64.7 \%)$ & $6(35.3 \%)$ & \\
\hline & Epirubicin & $11(36.7 \%)$ & $8(72.7 .0 \%)$ & $3(27.3 \%)$ & \\
\hline & Vinorelbine & $2(6.7 \%)$ & $2(100 \%)$ & $0(0.0 \%)$ & \\
\hline
\end{tabular}

Vascularity index $(\mathrm{VI})=$ the number of colored pixels within the tumor section/the number of total pixels in that particular tumor section.

* Hypervascularity: VI $>4.99 \%$ (median).

$\dagger$ Tumor with $3 \mathrm{~cm}$ in diameter, cut surface area $1.5 \mathrm{~cm} \times 1.5 \mathrm{~cm} \times \pi=7.06 \mathrm{~cm}$.

the initiation of neochemotherapy followed by decreasing vascularity (Fig. 3). We postulated that after initiation of chemotherapy, abundant angiogenic factors such as vascular endothelial growth factor (VEGF), released from apoptotic and necrotic breast cancer cells, resulted in profoundly increasing vascularity. After peak vascularity, the vascularity decreased gradually at a later stage of chemotherapy because of fewer angiogenic factors released by the remaining tumor cells that are chemotherapy sensitive. The time course survey of vascularity changes detected by power Doppler sonography (Fig. 3) did stop before the time of appearance for the final clinical outcome as described by Kedar et al. (1994) and Roberrto et al. (1998), which showed decreased tumor vascularity to the baseline correlated with improved clinical outcome. Instead, the key finding was that increased tumor vascularity appeared during early neoadjuvant chemotherapy. In addition, we found all patients with a VI increment of $>5 \%$ during chemotherapy had good chemotherapy response (i.e., 100\%) (Table 1). This amplitude of vascular change can be easily visible by the experienced sonographer, even without the aid of computation. We also observed in patients with a peak VI of $>10 \%$ during chemotherapy that, although the majority of their VI increment was $<5 \%$, the response rate was $94 \%$ (Table 1). A high vascularity profile during chemotherapy indicates good response to the treatment (Fig. 3, Tables 1, 2 and 3). These findings can be valuable surrogate markers in clinical practice based on two essential rationales.

Detection of chemotherapy response within one month based on vascularity profile makes power Doppler clinically applicable. Based on the angiogenic factorsrelated hypothesis (Furstenberger et al. 2006), we expected the angiogenic phase in $\mathrm{ABC}$ is an early event during chemotherapy. We found in patients with a VI increment of $>5 \%$ or a peak VI of $>10 \%$ after chemo- therapy, the time to reach the cutoff point is $25.47 \pm$ $12.96 \mathrm{~d}$ and $25.44 \pm 12.41 \mathrm{~d}$, respectively (Table 2 ). However, at these time points the tumor size reduction showed no statistical difference between responders and nonresponders (Table 4). This proved that the detectable vascularity change in Doppler sonography is a better indicator than the size reduction observed in B-mode ultrasonography.

There was no significance in response rate when they were grouped into hyper- and hypovascularity categories $(p=0.109)$ (Table 4). However, we noticed that mean initial VI for responders was $7.67 \pm 4.77 \%$ compared with the $4.01 \pm 3.82 \%$ in nonresponders. This difference has reached borderline significance $(p=$ 0.05). Thus, the initial VI of the tumor might be a useful indicator of the future response to chemotherapy (Table 5). This is contradictory to previous report (Boonjunwetwat et al. 2005). We proposed that hypervascular tumor provided more accessible vessels for receiving chemotherapy agents. Therefore, more tumor cells can readily contact with chemotherapy drug via surrounding blood vessels. However, the accessibility of tumors to the $\operatorname{drug}(\mathrm{s})$ is not the solely determining factor to chemotherapy response. In our unpublished data, we observed

Table 5. The difference in mean original VI between responders and nonresponders of neoadjuvant chemotherapy in thirty $\mathrm{ABC}$ patients

\begin{tabular}{lccc}
\hline \multicolumn{1}{c}{$\begin{array}{c}\text { Patient } \\
\text { responses }\end{array}$} & $\begin{array}{c}\text { No. of } \\
\text { patients }\end{array}$ & \multicolumn{2}{c}{ Original VI } \\
\hline $\begin{array}{l}\text { Responder } \\
\text { Nonresponder }\end{array}$ & 21 & $7.67 \pm 4.77 \%$ & $p=0.052$ \\
\hline
\end{tabular}

Vascularity index $(\mathrm{VI})=$ the number of colored pixels within the tumor section/the number of total pixels in that particular tumor section.

$\leqq$ VI decreased after chemotherapy 
that VEGF level surged after chemotherapy and fluctuated during the course of chemotherapy. This preliminary observation supported the finding that VEGF is a key coplayer in chemotherapy-induced neovascularity increment (Furstenberger et al. 2006). On the contrary, we found that VEGF was detected to be the basal level after surgery. This evidence favors the hypothesis that VEGF may come from tumor related factors either from the tumor itself or their supporting matrix, which was removed after operation (Furstenberger et al. 2006).

Notably, a recent study revealed that the combination of bevacizumab and Paclitaxel in previously untreated metastatic breast cancer (MBC) showed a statistically significant benefit in terms of overall response rate (28.2\% vs. $14.2 \%, p<0.0001)$, progression-free survival (PFS) (10.97 months vs. $6.11 \mathrm{mo}, p<0.001)$ and overall survival $(\mathrm{OS})(\mathrm{HR}=0.674, p=0.01)$, in favor of the combination arm (Miller et al. 2003). In our chemotherapy-responsive ABC patients, increasing vascularity is an early event during neoadjuvant chemotherapy. This observation may provide the insight, at least in part, that the combined cytotoxic and antiangiogenesis therapy has the synergistic effect on disease control of ABC. However, phase III studiess of chemotherapy plus bevacizumab in chemotherapy pretreated metastatic breast and colon cancer failed to show a benefit in these patients in terms of PFS and OS (Chen et al. 2004; Miller et al. 2005). Our surveillance showed a decreased vascularity at the later stage of chemotherapy. It is reasonable to expect that the tumor is less angiogenic-sensitive in the later course of chemotherapy. This observation supports the insignificant additive effect of antiangiogenic drug to chemotherapy in heavily treated metastatic cancers, which were likely chemotherapy resistant. Therefore, the effect of antiangiogenic drugs may be more prominent in the adjuvant setting than in the chemotherapy-pretreated tumors, which the angiogenic activities of tumors had diminished through pretreatment.

The major advantage of power Doppler sonography over other current methodologies in the field is for its efficiency in clinical vascularity determination. Low detection sensitivity is one of the most difficult problems of Doppler ultrasound. However, using power Doppler ultrasound, only a minimal frequency shift and a minimal number of intravascular reflecting particles are necessary for sufficient measurement and for increasing the sensitivity of detection (Taylor et al. 1988). Power Doppler ultrasound, in particular, is not angle dependent whenever a Doppler shift is detected. It is neither velocity dependent nor prone to aliasing (Kook et al. 1999; Milz et al. 2001; Raza and Baum 1997; Rubin et al. 1994). The power Doppler mode depicts the intensity or energy of the Doppler signals (integrated amplitude under spectral Doppler curve) for a time period. Power Doppler reduces the temporal solution and the visualization of vessel pulsatility. It does not show the direction or velocity of blood flow. But it depicts longer segments of smaller vessels as a result of holding the pixels colored for a longer time period. Therefore, it enables the examiner to get a more complex survey of intratumoral vascular structure than by conventional color Doppler. This is potentially useful, especially in breast cancer, because the vascular network of malignant tumors is characterized by a high capillary density of vessels, vessel-loops, arteriovenous shunts and chaotic vessel branching (Birdwell et al. 1997; Less et al. 1991; Martinoli et al. 1998; Milz et al. 2001; Weidner et al. 1991). Besides, power Doppler sonography has a significantly higher correlation with microvessel density when it is compared with 2-D color power Doppler sonography at baseline (Yang et al. 2002).

It is more sensitive in showing post-therapeutic changes of intratumoral blood flow by power Doppler sonography. Nevertheless, the role of power Doppler in changing therapeutic decisions has not been evaluated up to now despite its ability to improve the sensitivity in detection of blood flow compared with conventional color Doppler (Martinoli et al. 1998). Few papers address the correlation between Doppler ultrasound parameters and neoadjuvant chemotherapy response in breast cancer. Most of them are observational without any clinical predictive perspective. After two-point comparison of tumor vascularity before chemotherapy initiation and after surgery, all the papers concluded that decreased tumor vascularity indicates good response and increased or unchanged vascularity indicates no response at all (Kedar et al. 1994; Roberrto et al. 1998; Roubidoux et al. 2005). However, increasing vascularity was observed in three to four weeks after initiation of chemotherapy for frequent monitoring of tumor vascularity in a one- to two-week interval (Fig. 3), and it could not be observed by two-point comparison in previous reports. Furthermore, their methodologies were lack of quantitative measurement of Doppler signals, such as VI, which provides more objective comparison.

In neoadjuvant context, the traditional Doppler sonography parameters are less consistent and less informative. They include maximum flow velocity $\left(V_{\max }\right)$, pulsatility index (PI) and resistivity index (RI). First, the number of vessels, RI, PI and $V_{\max }$ fail to provide accurate differentiation of benign and malignant breast tumors (Chao et al. 1999). Second, in tumors with plentiful blood supply, vessel selection is highly subjective. In the pilot study, we observed the big variation of RI, PI and $V_{\max }$ of different vessels within a tumor (data not shown). Third, the impact of neovascularization after chemotherapy is hard to evaluate objectively with these conventional settings. 
From our study we found that quantitative power Doppler ultrasound is a useful tool in monitoring chemotherapy response of $\mathrm{ABC}$. Compared with other imaging modalities, computed tomography (CT) or dynamic magnetic resonance imaging (MRI) yielded a compound signal from blood flow, transcapillary diffusion and interstitial perfusion. It provided more sensitive imaging of increased vascularity, especially under contrast medium administration. Although MRI has superior sensitivity and more hemodynamic information, it is time consuming, less accessible and escalating in price. With slightly inferior precision, power Doppler ultrasound is cheaper, repeatable, readily available and has no radiation. It could be applied in a larger scale. However, it was examiner dependant, machine dependant and time consuming in the initial learning period. To avoid evaluation bias, this entire study was performed by a single investigator using the same ultrasound machine under consistent ultrasound settings.

In summary, neoadjuvant chemotherapy has been the standard treatment of $\mathrm{ABC}$. The number of patients with $\mathrm{ABC}$ at initial diagnosis is gradually decreasing because most of the breast cancer diagnosed today is detected in its early stage. $\mathrm{ABC}$ is a heterogeneous disease with a dismal problem in afflicted women, and the efficacy of chemotherapy for $\mathrm{ABC}$ is unpredictable. Power Doppler VI was an effective measure in monitoring response of neoadjuvant chemotherapy for $\mathrm{ABC}$ in this study. Given the complex biology underlying response to chemotherapy, one should not expect any single factor to be sufficiently predictive to guide therapy. In this preliminary study, in spite of small sample size and multiple regimens of chemotherapy, measurement of vascularity response to chemotherapy turned out to be an important strategy in breast cancer treatment, and this deserves further investigation in a larger patient population. Although this research finding was in our hands about five years ago, the solid scientific foundation of this finding was found recently by others only in an animal model (Shaked et al. 2006), and HDI 5000 with 12-MHZ linear probe of power Doppler ultrasound is still one of the most updated models worldwide. Therefore, we suggest the clinical application in the future to look for the differences and the parameters, which may influence the VI as well as the best time interval for measuring increased vascularity during neoadjuvant chemotherapy upon updated treatment protocols and more recent advanced tools applied.

\section{CONCLUSION}

We propose that the VI, as determined by using power Doppler sonography, is a good and inexpensive clinical tool for monitoring vascularity changes during neoadjuvant chemotherapy in ABC patients. Two parameters-VI increment $>5 \%$ and peak VI $>10 \%$-are potential early predictors for good responses to neoadjuvant chemotherapy within one month in patients with $\mathrm{ABC}$.

Acknowledgements-This study was supported by funds from National Science Council in Taiwan: NSC 95 to $2811-B-002$ to 009 , NSC 95 to 2622-B-002 to 274 (to K.J.C.); NSC95-2314-B-002 to 255- MY3(to F.J.H); by grants from the Department of Industrial Technology, Ministry of Economic Affairs, Taipei, Taiwan (to K.J.C.); and the Foundation of Breast Cancer Prevention and Treatment, Taiwan (to K.J.C.).

\section{REFERENCES}

Birdwell RL, Ikeda DM, Jeffrey SS, Jeffrey RB Jr. Preliminary experience with power Doppler imaging of solid breast masses. Am J Roentgenol 1997;169:703-707.

Bonadonna G, Valagussa P, Brambilla C, Moliterni A, Zambetti M, Ferrari L. Adjuvant and neoadjuvant treatment of breast cancers with chemotherapy and endocrine therapy. Semin Oncol 1991;18: 515-524.

Boonjunwetwat D, Prueksadee J, Sampatanukul P, Chatamra K. Does color Doppler ultrasound vascularity predict the response to neoadjuvant chemotherapy in breast cancer? J Med Assoc Thai 2005; 88(10):1367-1372.

Caruso G, Cucciarre S, Lo Bello M, Mercurio G, Candela F, Cianchino C, Lagalla R. Neoadjuvant intralesional chemotherapy for breast neoplasm in advanced stage. Assessment of its efficacy with Doppler color. Radiol Med (Torino) 1995;89(5):613-618.

Chao TC, Lo YF, Chen SC, Chen MF. Color Doppler ultrasound in benign and malignant breast tumors. Breast Cancer Res Treat 1999;57(2):193-199.

Chen CN, Cheng YM, Liang JT, Lee PH, Hsieh FJ, Yuan RH, Wang SM, Chang MF, Chang KJ. Color Doppler vascularity index can predict distant metastasis and survival in colon cancer patients. Cancer Res 2000;60:2892-2897.

Chen HX, Mooney M, Boron M, Vena D, Mosby K, Grochow L, Jaffe C, Rubinstein L, Zwiebel J, Kaplan RS. Bevacizumab plus 5-FU/ leucovorin for advanced colorectal cancer that progressed after standard chemotherapies: An NCI Treatment Referral Center Trial (TRC-0301). J Clin Oncol 2004;22(14S):248s.

Cheng WF, Lee CN, Chu JS, Chen CA, Chen TM, Shau WY, Hsieh CY, Hsieh FJ. Vascularity index as a novel parameter for the in vivo assessment of angiogenesis in patients with cervical carcinoma. Cancer (Phila) 1999;85:651-657.

Clahsen PC, van de Velde CJ, Julien JP, Floiras JL, Delozier T, Mignolet FY, Sahmoud TM. Improved local control and diseasefree survival after perioperative chemotherapy for early-stage breast cancer. A European Organization for Research and Treatment of Cancer Breast Cancer Cooperative Group Study. J Clin Oncol 1996;14(3):745-753.

Cosgrove DO, Bamber JC, Davey JB, McKinna JA, Sinnett HD. Color Doppler signals from breast tumors. Work in progress. Radiology 1990;176:175-180.

Cosgrove DO, Kedar RP, Bamber JC, al-Murrani B, Davey JB, Fisher C, McKinna JA, Svensson WE, Tohno E, Vagios E. Breast diseases: Color Doppler US in differential diagnosis. Radiology 1993; 189:99-104.

Dixon JM, Walsh J, Paterson D, Chetty U. Color Doppler ultrasonography studies of benign and malignant breast lesions. Br J Surg 1992;79:259-260.

Folkman J, Long D, Becker F. Growth and metastasis of tumor in organ culture. Cancer (Phila) 1963;16:453-457.

Furstenberger G, von Moos R, Lucas R, Thurlimann B, Senn HJ, Hamacher J, Boneberg EM. Circulating endothelial cells and angiogenic serum factors during neoadjuvant chemotherapy of primary breast cancer. Br J Cancer 2006;94(4):524-531.

Hayward JL, Carbone PP, Heusen JC, Kumaoka S, Segaloff A, Rubens $\mathrm{RD}$. Assessment of response to therapy in advanced breast cancer. Br J Cancer 1977;35(3):292-298. 
Hsu C, Chen CN, Chen LT, Wu CY, Hsieh FJ, Cheng AL. Effect of thalidomide in hepatocellular carcinoma: Assessment with power Doppler US and analysis of circulating angiogenic factors. Radiology 2005;235(2):509-516.

Huber S, Medl M, Helbich T, Taucher S, Wagner T, Rudas M, Zuna I, Delorme S. Locally advanced breast carcinoma: Computer assisted semiquantitative analysis of color Doppler ultrasonography in the evaluation of tumor response to neoadjuvant chemotherapy (work in progress). J Ultrasound Med 2000;19:601-607.

Kedar RP, Cosgrove DO, Smith IE, Mansi JL, Bamber JC. Breast carcinoma: Measurement of tumor response to primary medical therapy with color Doppler flow imaging. Radiology 1994;190: 825-830.

Kook SH, Park HW, Lee YR, Lee YU, Pae WK, Park YL. Evaluation of solid breast tumors with power Doppler sonography. J Clin Ultrasound 1999;27:231-237.

Lagalla R, Caruso G, Finazzo M. Monitoring treatment response with color and power Doppler. Eur J Radiol 1998;27 Suppl 2:S149-56.

Less JR, Skalak TC, Sevick EM, Jain RK. Microvascular architecture in a mammary carcinoma: Branching patterns and vessel dimensions. Cancer Res 1991;51:265-273.

Mamounas EP. Overview of National Surgical Adjuvant Breast Project neoadjuvant chemotherapy studies. Sem Oncol 1998;(Suppl 3): 31-35.

Martinoli C, Pretolesi F, Crespi G, Bianchi S, Gandolfo N, Valle M, Derchi LE. Power Doppler sonography: Clinical applications. Eur J Radiol 1998;27(Suppl 2):S133-S140.

Miller KD. E2100: A phase III trial of paclitaxel versus paclitaxel/ bevacizumab for metastatic breast cancer. Clin Breast Cancer 2003; 3(6):421-422.

Miller KD, Chap LI, Holmes FA, Cobleigh MA, Marcom PK, Fehrenbacher L, Dickler M, Overmoyer BA, Reimann JD, Sing AP, Langmuir V, Rugo HS. Randomized phase III trial of capecitabine compared with bevacizumab plus capecitabine in patients with previously treated metastatic breast cancer. J Clin Oncol 2005;23: 792-799.

Milz P, Lienemann A, Kessler M, Reiser M. Evaluation of breast tumors by power Doppler sonography. Eur Radiol 2001;11:547554.

Raza S, Baum JK. Solid breast tumors: Evaluation with power Doppler US. Radiology 1997;203:164-168.

Roberrto L, Giuseppe C, Mario F. Monitoring treatment response with color and power Doppler. Eur J Radiol 1998;28 suppl:S149-156.

Roubidoux MA, LeCarpentier GL, Fowlkes JB, Bartz B, Pai D, Gordon SP, Schott AF, Johnson TD, Carson PL. Sonographic evaluation of early-stage breast cancers that undergo neoadjuvant chemotherapy. J Ultrasound Med 2005;24:885-895.

Rubin JM, Bude RO, Carson PL, Bree RL, Adler RS. Power Doppler US: A potentially useful alternative to mean frequency-based color Doppler US. Radiology 1994;190:853-856.

Shaked Y, Ciarrocchi A, Franco M, Lee CR, Man S, Cheung AM, Hicklin DJ, Chaplin D, Foster FS, Benezra R, Kerbel RS. Therapyinduced acute recruitment of circulating endothelial progenitor cells to tumors. Science 2006;313(5794):1785-1787.

Singh S, Pradhan S, Shukla RC, Ansari MA, Kumar A. Color Doppler ultrasound as an objective assessment tool for chemotherapeutic response in advanced breast cancer. Breast Cancer 2005;12(1): 45-51.

Smith IC, Heys SD, Hutcheon AW, Miller ID, Payne S, Gilbert FJ, Ah-See AK, Eremin O, Walker LG, Sarkar TK, Eggleton SP, Ogston KN. Neoadjuvant chemotherapy in breast cancer: Significantly enhanced response with docetaxel. J Clin Oncol 2002;20(6): $1456-1466$

Smith IE. Primary (neoadjuvant) medical therapy. In: Powles TJ, Smith IE, eds. Medical Management of Breast Cancer. London: Martin Dunitz, 1991: 259-265.

Taylor KJ, Ramos I, Carter D, Morse SS, Snower D, Fortune K. Correlation of Doppler US tumor signals with neovascular morphologic features. Radiology 1988;166(1 Pt 1):57-62.

Toi M, Kashitani L, Tominaga T. Tumor angiogenesis is an independent prognostic indicator in primary breast carcinoma. Int J Cancer 1993;55:371-374.

Vallone P, D'Angelo R, Filice S, Petrosino T, Rinaldo M, De Chiara A, Gallipoli A. Color-doppler using contrast medium in evaluating the response to neoadjuvant treatment in patients with locally advanced breast carcinoma. Anticancer Res 2005;25:595-599.

Weidner N, Semple JP, Welch WR, Folkman J. Tumor angiogenesis and metastasis-correlation in invasive breast carcinoma. N Engl J Med 1991;324:1-8.

Weidner N, Folkman J, Pozza F, Bevilacqua P, Allred EN, Moore DH, Meli S, Gasparini G. Turmor angiogenesis: A new significant and independent prognostic indicator in early stage breast carcinoma. J Nat Cancer Inst 1992;84:1875-1887.

Wu CH, Hsu MM, Chang YL, Hsieh FJ. Vascular pathology of malignant cervical lymphadenopathy. Cancer (Phila) 1998;83:11891196.

Yang WT, Tse GM, Lam PK, Metreweli C, Chang J. Correlation between color power Doppler sonographic measurement of breast tumor vasculature and immunohistochemical analysis of microvessel density for the quantitation of angiogenesis. J Ultrasound Med 2002;21:1227-1235. 